\title{
Le FLS : un concept en question
}

Jean-Pierre Cuq

\section{OpenEdition}

Journals

Édition électronique

URL : http://journals.openedition.org/trema/2153

DOI : 10.4000/trema.2153

ISSN : 2107-0997

\section{Éditeur}

Faculté d'Éducation de l'université de Montpellier

\section{Édition imprimée}

Date de publication : 1 juin 1995

Pagination : 3-11

ISSN : 1167-315X

\section{Référence électronique}

Jean-Pierre Cuq, «Le FLS : un concept en question», Tréma [En ligne], 7 | 1995, mis en ligne le 23 septembre 2013, consulté le 19 avril 2019. URL : http://journals.openedition.org/trema/2153 ; DOI : 10.4000/trema.2153

Ce document a été généré automatiquement le 19 avril 2019

Trema 


\title{
Le FLS : un concept en question
}

\author{
Jean-Pierre Cuq
}

1 Français langue seconde, français langue étrangère, français langue maternelle, français pour migrants... et combien d'autres appellations encore pour notre idiome commun! Comment un étudiant, un enseignant, éclairé certes, mais pas forcément spécialiste de ces questions peut-il se retrouver dans un tel foisonnement terminologique? Sans compter les sigles: FLS, FLE, FLM... Du reste, s'agit-il d'une simple question terminologique aux paramètres plus ou moins bien maitrisés ? N'y a-t-il pas là plutôt quelque problème conceptuel plus difficile à appréhender? Dans ce cas, dans quel champ disciplinaire se situent les concepts concernés (sociolinguistique, didactique...) ?

2 Voilà quelques questions, avec quelques autres, auxquelles j'avais tenté de répondre en 1991 dans Le français langue seconde ${ }^{1}$. Dans la conclusion de l'ouvrage, je confiais ce bateau un peu frêle aux courants didactiques: il toucherait les ports qui voudraient bien l'accueillir. L'année suivante, au Congrès de la Fédération internationale des professeurs de français de Lausanne, cette étude s'est augmentée d'un état des lieux plus complet de la recherche en français langue seconde ${ }^{2}$. Ces deux publications, dont les données n'ont, pour l'essentiel, pas encore trop vieilli, vont donc servir de base à cette réflexion.

Il semble qu'il ne soit pas tout-à-fait inutile de revenir sur certains points, soit qu'ils aient été mal développés à l'époque, soit qu'ils aient été depuis sujets à polémique, soit enfin qu'à propos de certains ma propre position ait un peu évolué.

4 L'ouverture de ce numéro de TREMA va permettre de relancer le débat. Mon propos s'articulera autour des trois questions suivantes: tout d'abord, qu'est-ce que le français langue seconde (FLS)? Dans cette première partie seront abordés les questions terminologiques, les thèmes de notion et de concept, le positionnement épistémologique. Ensuite, la France métropolitaine est-elle concernée par cette notion? On examinera alors la question du français pour migrants. Enfin, le français langue seconde est-il utile? Ce concept n'est-il pas sensible, c'est-à-dire dépassant les simples enjeux didactiques? 


\section{Qu'est-ce que le français langue seconde?}

5 Voici la définition que j'en proposais en $1991^{3}$ :

«Le français langue seconde est un concept ressortissant aux concepts de langue et de français. Sur chacune des aires où il trouve son application, c'est une langue de nature étrangère. Il se distingue des autres langues étrangères éventuellement présentes sur ces aires par ses valeurs statutaires, soit juridiquement, soit socialement, soit les deux, et par le degré d'appropriation que la communauté qui l'utilise s'est octroyé ou revendiqué. Cette communauté est bi ou plurilingue. La plupart de ses membres le sont aussi et le français joue dans leur développement psychologique, cognitif et informatif, conjointement avec une ou plusieurs autres langues, un rôle privilégié. »

6 Il s'agit d'un concept, c'est-à-dire d'une construction abstraite, destinée à catégoriser le réel auquel elle renvoie. Pierre Martínez a eu raison de remarquer que le titre de la deuxième partie du livre ("Les fondements de la notion de français langue seconde ») est mauvais. En effet, une notion est la connaissance intuitive qu'on peut avoir du réel (exemple : la notion de chaleur). Or le français langue seconde n'est pas une connaissance intuitive, il est une théorisation à partir de l'intuition qu'il existe des similitudes entre une série de situations distinctes d'appropriation du français. A ce titre, il est forcément un peu réducteur, mais toutes les situations visées doivent présenter une série de paramètres stables, qu'il décrit, et une série de variables qu'il ne prend pas en compte. Or c'est justement très souvent une perception erronée de ces variables qui, tel l'arbre cachant la forêt, ont longtemps empêché l'émergence de ce concept.

7 Il s'agit d'un concept didactique et non pas linguistique. Personne ne parle le français langue seconde, pas plus d'ailleurs que le français langue étrangère (FLE) ou le français langue maternelle (FLM). On parle, on apprend, on acquiert un idiome qui a pour nom français, pluriel dans ses variations observables, bien qu'il soit fortement unifié par une norme écrite à tendance centralisatrice. La question didactique est donc à situer dans les moyens à mettre en œuvre pour que des individus s'approprient au mieux cet idiome. La définition d'un concept fait partie de cette intervention. Pour y parvenir, il est bien entendu nécessaire d'interroger des disciplines plus ou moins voisines: la linguistique (état des variations répertoriées, notion de la langue même, qui est contenue dans l'item FLS), la sociolinguistique (qui sont les locuteurs concernés), l'histoire (pour quelles raisons sont-ils concernés? quelles sont les forces idéologiques en présence?), la géographie (quelles sont les conditions humaines locales?), l'économie, etc. Mais le résultat est une abstraction didactique, c'est-à-dire qui prend sa valeur dans un environnement conceptuel didactique, et particulièrement dans l'opposition avec les concepts de langue maternelle et de langue étrangère.

En ce qui concerne le français langue maternelle, on peut considérer que cet item est une facilité de langage ${ }^{4}$. Il est en effet un peu trop centré sur la transmission de la langue par la seule mère, alors qu'on connaît de très nombreux contre-exemples, y compris au niveau de sociétés. On préférera donc utiliser langue première, ou langue de première socialisation, ou mieux, langue de départ, c'est-à-dire, le premier (ou les premiers) idiome (s) dans le(s)quel(s) l'enfant se construit. Un autre aspect très important qui n'est pas suffisamment pris en compte est la langue d'appartenance, ou langue revendiquée, "qui correspond à l'ensemble des attitudes et des représentations d'un sujet ou d'un groupe face à la langue en tant qu'élément constitutif de l'identité $»^{5}$ Un des problèmes posés par 
le FLS est justement l'ambiguïté qui existe souvent, chez les locuteurs ou les groupes sociaux concernés, entre le sentiment d'un positionnement différent de celui des autres locuteurs étrangers face au français, et le sentiment des risques identitaires et culturels que peut faire encourir l'acquisition d'un idiome qui a pris une valeur privilégiée dans leur communauté. D'où également, une certaine résistance à l'adjectif seconde dans l'expression français langue seconde, qui peut apparaître de ce point de vue comme agressif.

Malgré tous les inconvénients que présente son nom, on conservera l'étiquette langue seconde pour le concept qui nous occupe, en réaffirmant fortement qu'elle demeure une sous-catégorisation du FLE. D'abord, parce qu'est étrangère pour l'individu toute langue autre que sa langue de départ, même si, ayant une réalité juridique ou sociale dans son pays, elle n'est pas toujours considérée comme telle au niveau national. Cela fait ensuite qu'elle pose des problèmes d'enseignement et d'apprentissage qui, même s'ils sont spécifiques et justifient la prise en compte de paramètres qui n'ont que peu de poids dans les autres situations de FLE, sont d'une nature de FLE et non d'une nature intermédiaire entre le FLM et le FLE.

Comment dès lors situer le cas des étrangers venus s'établir définitivement en pays où le français est la langue première de la majeure partie de la population? C'est dont nous allons débattre maintenant.

\section{La France métropolitaine ${ }^{6}$ et le français langue seconde}

11 La deuxième partie du Français langue seconde (op. cit.), expliquait en 1991 pourquoi le FLS se distingue du français pour migrants. L'argument principal est qu'un migrant, contrairement aux autres individus ou groupes en situation de langue seconde, ne se situe pas dans une logique d'intégration à un groupe dont la ou les langues premières ne sont pas le français. Tous les autres parleurs seconds de français se situent en effet dans une dynamique de bi ou de plurilinguisme. Or on constate au contraire qu'en France métropolitaine, la dynamique intégrative est très largement monolingue. Cela s'explique par le fait que sur le territoire métropolitain le français est la langue dominante du point de vue du nombre, du point de vue politique et du point de vue culturel. Même s'il ne saurait être question sur un territoire aussi vaste et aussi ouvert de parler, au sens sociolinguistique du terme, de monolinguisme, il n'en demeure pas moins incontestable que tous les autres idiomes éventuellement concurrents se trouvent en position totalement dominée. En tenant cette position, je n'ignorais évidemment pas qu'il ne pouvait s'agir que d'une généralisation. On constate en effet des différences fortes chez les individus en fonction de leur milieu social, de leur région géographique d'origine, de leur âge et de leur sexe. J'avais pris également soin de préciser qu'il ne s'agissait pas dans mon esprit d'affirmer que ce passage d'un monolinguisme en langue étrangère à un monolinguisme en français se faisait obligatoirement au cours de la même génération, c'est-à-dire dans la vie d'un seul individu, mais qu'il se produisait sur deux, voire trois générations, et qu'il restait très souvent des traces familiales plus ou moins importantes de l'idiome initial. Prenons pour exemple l'immigration polonaise dans la ville minière de La Mure, près de Grenoble. Il serait évidemment très intéressant de disposer d'une étude 
sociolinguistique précise sur la question, qui pourrait confirmer les faits suivants constatés dans certaines familles :

- les parleurs de première génération sont bilingues avec polonais régressif mais largement interférant ;

- les parleurs de deuxième génération sont bilingues avec français dominant et des compétences incomplètes en polonais : en règle générale, faute sans doute de scolarisation ${ }^{7}$ en cette langue, ce sont les compétences d'écrit (écriture et lecture) qui disparaissent les premières, la compréhension orale demeurant le plus longtemps la plus active ;

- les parleurs de troisième génération gardent pour certains une certaine compréhension orale mais surtout des expressions idiomatiques qui viennent « colorer » leur français.

Naturellement, tout ceci me paraît être une tendance, qui peut toujours se trouver plus ou moins infirmée par des faits individuels plus typés.

Mais pourquoi cette position est-elle controversée?

D'abord, la plupart des auteurs anglo-saxons (et on connaît le poids de la sociolinguistique anglo-saxonne) admettent les situations de migration comme faisant partie de l'anglais langue seconde (English as second language) ${ }^{8}$. J'avais souligné ce fait en 1991, et les recensions anglo-saxonnes de mon travail ont relevé ma prise de position comme une curiosité, sans d'ailleurs y trouver particulièrement à redire.

D'autre part, beaucoup de didacticiens intéressés par le FLS viennent des rangs de ceux qui s'occupent ou se sont occupés des questions scolaires et linguistiques liées à la migration en France.

Mais la critique la plus dure est venue d'auteurs francophones. Mwatha Ngalasso ${ }^{9}$ trouve cette position "surprenante " et "guère convaincante » puisque ce monolinguisme prévisible n'interviendra qu'après plusieurs générations et que les sujets ne pourront plus alors être qualifiés de migrants. Cette objection est forte. Loin de la minimiser, je peux même m'y rallier en partie. Avant cela, on me permettra en revanche de passer assez vite sur le second argument avancé par Ngalasso sous forme de question: "Comment considérer la situation du Polonais, du Portugais, du Sierra-Léonais ou même du Sénégalais qui a appris ou non un peu de français dans son pays et qui, parce qu'il désire travailler ou poursuivre en français, ses études en France ou en Belgique, s'engage, sans risquer de perdre sa langue maternelle, à un apprentissage de la langue française ?» Il y a en effet à cette question une réponse très simple: c'est une situation de français langue étrangère banale, dite d'apprentissage endolingue. On retiendra tout de même de cet argument l'accent mis sur la situation d'apprentissage/enseignement parce qu'il s'agit justement d'un paramètre dont je croyais avoir suffisamment montré l'insuffisance lorsqu'il est avancé seul.

Mais revenons à l'objection forte. La question qui se pose en effet est de savoir si, au cours de la vie seule d'un individu, celui-ci peut se trouver, en cas de migration, en position d'apprentissage du français comme langue seconde. J'admettrai donc, dans ce cas, le peu de pertinence de l'argumentation qui consiste à laisser dérouler sur deux ou trois générations le processus d'assimilation. On sait pourtant qu'il demeure souvent, et d'ailleurs surtout à la troisième génération, une revendication identitaire qui va parfois jusqu'à faire réapprendre la langue d'origine familiale pour retrouver grâce à elle un certain enracinement culturel. On ne peut certes plus qualifier ces individus d'immigrés, mais on peut peut-être voir chez eux une certaine particularité comparable, toutes choses égales par ailleurs, à la revendication d'un régionalisme, qui aurait ceci de particulier, qu'il serait privé de son terroir. De plus, comme je l'ai évoqué plus haut, la situation des 
langues en présence dans ces cas est totalement déséquilibrée en faveur du français, la langue première de l'apprenant ne jouant plus vraiment de rôle hors de la cellule familiale, ce qui n'est pas le cas dans les situations de FLS proprement dit. Reste donc l'aspect méthodologique de la question, c'est-à-dire la question de savoir si on peut mettre en œuvre pour les migrants une méthodologie de type FLS ou une méthodologie en partie différente. Il est bien possible que la réponse à cette question soit oui, mais ce n'est pas démontré. En tout état de cause, s'il existe une différence entre ce qui serait deux concepts séparés, on peut penser qu'elle est petite (sans avoir mené son élaboration jusqu'à son terme, ce que ne me permettrait pas ma médiocre compétence en matière de français pour migrants, j'ai donné plus haut quelques pistes). Il n'est donc pas anormal que les différences entre les conséquences méthodologiques soient petites aussi. Ainsi, et à titre de comparaison, peut-on voir que les méthodologies à mettre en œuvre en FLE et en FLS présentent elles aussi des différences mais qu'elles ont, comme il est normal pour des concepts en inclusion, bien des similitudes.

Il se peut donc que faute de disposer d'un bon outillage conceptuel pour le français pour migrants, on ait (provisoirement ?) utilisé le concept de $\mathrm{FLS}^{10}$. Au bout du compte donc, il est bien possible qu'on ait affaire là à une attitude d'approximation conceptuelle et que, pour beaucoup, les auteurs n'en jugent qu'à partir d'un certain empirisme méthodologique, voire pédagogique. Or en didactique des langues pas plus que dans les autres domaines scientifiques, la méthodologie ne se situe pas au même niveau que la conceptualisation, et encore moins la pédagogie, qui dépend de la méthodologie ${ }^{11}$. Mon opinion est donc qu'il faut se garder a priori des apparences. Rien ne dit en effet que le fait qu'il puisse exister des pratiques pédagogiques communes renvoie à une communauté conceptuelle, et il me parait tout à fait concevable au contraire qu'un travail d'élaboration au niveau conceptuel, pour le français pour migrants, puisse conduire à une pratique pédagogique mieux banalisée sinon complètement rénovée.

Mais il me semble qu'en fait cette discussion sur un point somme toute mineur fait ressortir le problème de fond suivant.

\section{Le concept de langue seconde est-il utile en didactique des langues?}

Parmi les reproches qui ont été adressés au concept de FLS on en trouve plusieurs assez faciles à réfuter mais difficiles à supprimer des débats car ils renvoient, me semble-t-il, à une certaine mauvaise volonté de lecture, voire au procès d'intention. J'en prendrai quelques exemples.

19 Le premier consiste à jouer sur l'adjectif second pour indiquer que le français ne saurait être second dans les cas, assez fréquents en Afrique par exemple, où l'apprenant a déjà deux langues ou plus dans son répertoire verbal avant de commencer l'apprentissage du français. D'abord, second indiquant en français une fin de série, on aurait plutôt employé dans ce cas deuxième. Ensuite, et plus sérieusement, l'ordre d'acquisition linguistique est un facteur peut-être peu pertinent pour établir le concept et j'avais moi-même souligné la malheureuse ambiguïté de l'item FLS, dont la paternité n'est à vrai dire pas très bien établie. seulement institutionnelle et à réfuter pour cela l'idée selon laquelle le français est 
langue de scolarisation. On donne à cet effet des exemples de pays dans lesquels le français n'est pas la seule langue de scolarisation proposée (ex: Zaïre, Cameroun), ou dans lesquels il le devient très tardivement et dans certaines disciplines seulement (ex : Maroc). Or dans la définition que j'avais proposée en 1991 je m'étais bien gardé d'affirmer cela. J'avais retenu une formule plus modérée : « ... le français joue dans leur (i.e., celui des membres des sociétés concernées) développement psychologique, cognitif et informatif, conjointement avec une ou plusieurs autres langues, un rôle privilégié.» On voit bien que ce n'est pas l'aspect institutionnel que j'avais retenu dans cette partie de la définition mais au contraire l'aspect cognitif. Quant à l'argument subséquent, qui consiste à laisser entendre qu'un enseignement en langue non maternelle est nuisible au bon développement psychologique de l'enfant, personne n'est en mesure d'affirmer aujourd'hui sa validité. Plusieurs études menées par exemple au Canada sur des élèves qui ont suivi des cursus d'immersion sembleraient même montrer le contraire ${ }^{12}$. Avancer un tel argument est donc, plutôt qu'une position didactique, une prise de position idéologique, ce que je ne conteste pas à condition de l'affirmer comme telle. En effet, on connaît bien l'enjeu que représente la scolarisation en langue non maternelle, et en particulier dans la pérennisation de situations linguistiques héritées de la colonisation. Or il se trouve qu'en Afrique, plusieurs expériences de scolarisation en langue locale ont malheureusement conduit à des échecs ou à des résultats pour le moins mitigés. Ce qui ne condamne pas cette position de manière rédhibitoire, car il est possible que les conditions faites aux expériences aient largement été la cause de leur échec. Je pense ici à la Guinée de Sékou Touré par exemple, mais on pourrait aussi évaluer les politiques d'arabisation, souvent présentées comme un retour à la langue maternelle, ce qui n'a aucun fondement linguistique, l'arabe classique n'ayant jamais été la langue maternelle de personne mais une langue revendiquée: leurs résultats semblent être, compte tenu des difficultés de l'entreprise, relativement convenables en Tunisie et au Maroc, mauvais en Algérie ${ }^{13}$ Quoi qu'il en soit, et pour ce qui me concerne, j'ai toujours affirmé que les choix d'aménagement linguistique ne sont pas du ressort du didacticien, surtout lorsqu'il est étranger. Son intervention commence, sauf lorsqu'on lui demande des études de conseil, une fois que les décisions politiques sont prises par les responsables des pays concernés. De fait, le statut des langues est par nature évolutif. Nul ne prétend que la situation qui est aujourd'hui celle du français sera la sienne demain : c'est à chaque Etat d'en décider et sans doute aussi, qu'il le veuille ou non, qu'il le sache ou non, à chaque citoyen.

Plus lourd encore de conséquence me parait être l'argument qui consiste à affirmer que l'hétérogénéité des terrains d'application engendre des difficultés à chaque fois particulières et qu'il est donc peu utile de disposer d'un outillage conceptuel transversal. Je ferai tout d'abord remarquer que le même argument pourrait être avancé à l'encontre du FLE. Qu'y a-t-il en effet de commun dans l'enseignement du français, disons, par exemple, à de jeunes lycéens en Moldavie et à des adultes venus individuellement en France de plusieurs pays et qui se retrouvent dans une même classe dans un centre de langue française? Tout a priori paraît séparer les deux cas: situation endolingue vs exolingue, adolescents vs adultes, groupes homogènes vs hétérogènes, professeur natif vs non natif, sans parler des différences éventuelles de motivation et des moyens technologiques méthodologiques etc. qui peuvent être mis en œuvre. Or il ne vient plus aujourd'hui à l'idée de personne de réfuter l'idée qu'il s'agit tout de même dans les deux cas de français langue étrangère et que ceci est à distinguer des situations de français langue maternelle. En revanche chacun des paramètres que j'ai évoqués justifie sans doute un affinage conceptuel ou méthodologique, mais cela ne vient qu'à un deuxième 
niveau. Il en est de même pour le FLS : refuser le concept sous le prétexte des différences de terrain, c'est revenir à la situation qu'on connaissait dans les années quatre-vingt et qui m'a poussé, comme quelques autres, à m'interroger non pas sur les différences, mais au contraire sur les similitudes existant entre toutes les situations que je connaissais ou qu'on évoquait avec moi. Or cette attitude de refus a deux corollaires. Le premier est une absence d'action méthodologique spécifique : si tout est égal à tout et réciproquement, pourquoi tenter une action différenciée ? Le second corollaire est un simple recours à l'empirisme pédagogique, c'est-à-dire in fine au refus d'une attitude scientifique face aux situations d'enseignement/apprentissage. Or c'est justement le droit de se prévaloir d'une attitude scientifique (beaucoup plus que d'établir une nouvelle science) que réclament les didacticiens face à ceux qui réfutent la légitimité de leur discipline. Je n'avance cet argument qu'à titre de mise en garde épistémologique car j'admettrai ici bien volontiers que la discussion, en revanche, est tout à fait indispensable au progrès de la réflexion.

\section{Conclusion}

Ces quelques pages paraitront à beaucoup être un plaidoyer plus qu'une réflexion. C'est qu'il me paraissait opportun de faire état, dans cette présentation, de plusieurs réactions suscitées ces dernières années par l'émergence du concept de FLS. Beaucoup d'arguments qui lui sont opposés sont peu convaincants, et résultent souvent plus d'anciens réflexes ou de positions de type idéologique que de type didactique. Mais, comme le montre le thème du présent numéro, la discussion est heureusement loin d'être close.

Après quelques années d'enseignement et de formation dans les pays où il me semblait avoir rencontré une situation didactique spécifique, il m'avait paru naturel de tenter une certaine théorisation des pratiques méthodologiques et pédagogiques rencontrées. L'outillage conceptuel et méthodologique alors disponible ne me paraissait pas suffisant pour affronter mes tâches quotidiennes correctement. Mais, si en didactique la réflexion conceptuelle peut être menée de n'importe où, à condition qu'elle ait été nourrie d'une certaine expérience pratique, l'action méthodologique et pédagogique appartient prioritairement à ceux qui vivent les situations d'enseignement sur le terrain. C'est donc à tous les collègues concernés par les situations rassemblées sous la dénomination de FLS qu'est aujourd'hui confié l'avenir de ce concept. Il est sans doute encore trop tôt aujourd'hui pour apprécier son influence éventuelle. Espérons au moins qu'il aura servi à banaliser un moment la réflexion sur ce qui demeure l'enjeu majeur du siècle à venir pour notre langue : la réussite ou l'échec de son enseignement à des populations qui sont devenues dès aujourd'hui dans des conditions si particulières, la majorité des francophones. 


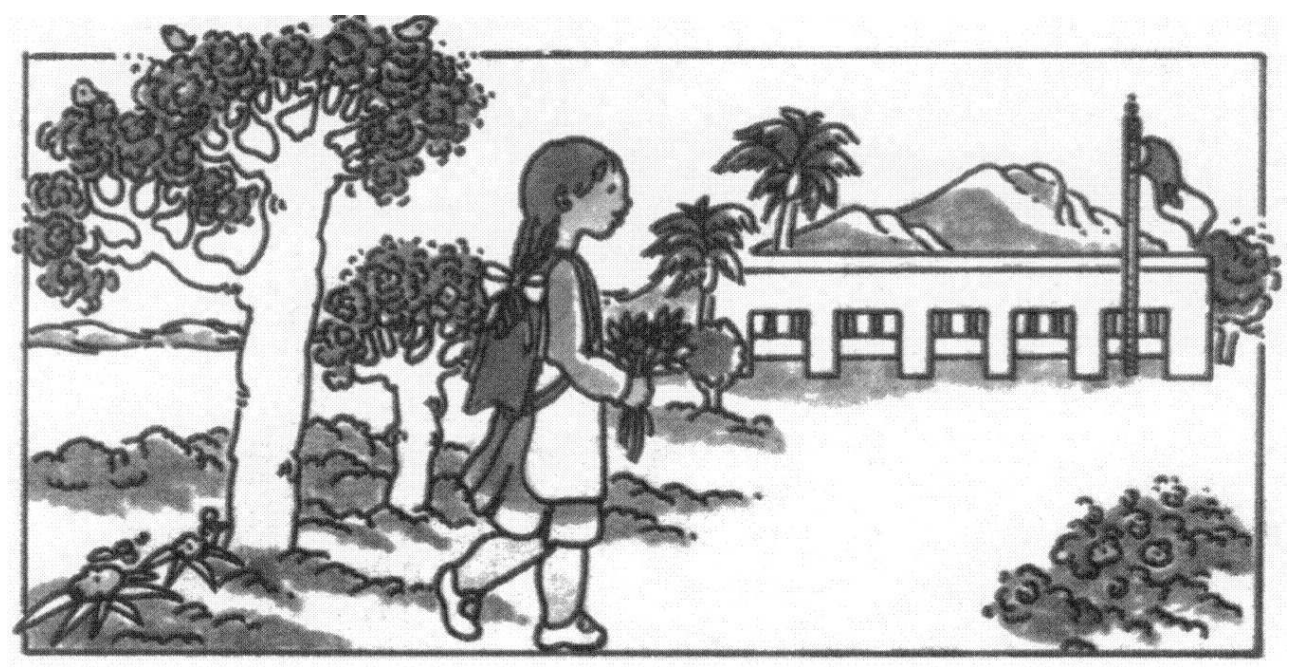

\section{NOTES}

1. Cuq J.-P, Le français langue seconde. Hachette, $\mathrm{F}, 1991$.

2. «Français langue seconde. Un point sur la question. » Etudes de linguistique appliquée, numéro spécial, 88, 1992. pp. 5-26.

3. Le français langue seconde. Hachette, F, 1991. p. 139.

4. Voir par exemple. Dabène L. Repères sociolinguistiques pour l'enseignement des langues. Hachette, F, 1994.

5. Dabène L, op. cit. p. 27.

6. La situation dans les DOM-TOM est toute différente, c'est pourquoi je précise par métropolitaine.

7. Toutefois, une maîtresse de polonais a donné des leçons aux enfants de familles immigrées jusqu'à la fin des années 1960. Elle insistait surtout sur la pratique orale. On peut voir là une pratique privée, et en avance sur son temps, de ce que l'école publique appellera plus tard l'enseignement des langues et cultures d'origine (LCO).

8. Par exemple Nicholls et Naish, Teaching English as a Second Language, British Broadcasting Corporation, 1981 ; Kennedy. « The Future of English Language Teaching », dans System, 14, 1986.

9. "Le concept de français langue seconde ", dans Etudes de linguistique appliquée, numéro spécial, 88, 1992, p. 31.

10. Cette raison rejoint d'ailleurs la première. La langue anglaise, même si elle a connu, en volume, une extension bien plus grande que le français, est du même type en ce qui concerne son développement: c'est une lingua franca, répandue sur les cinq continents par émigration, colonisation puis adoption, et sujette aujourd'hui à une attraction de type migratoire tant sur son aire originelle (Angleterre) que sur certaines aires naguère d'immigration ou de colonisation (Etats-Unis. Australie ... ).

11. Voir sur ce point J.-P. Cuq: «La conceptualisation, un moment central de l'intervention didactique en français langue étrangère ", dans TRA.DI.FLE, 30, 1993. 
12. Entre autres Lapkin S. et Swain M. : « Faisons le point » dans Langue et Société. $\mathrm{n}^{\circ}$ 12, 1984, pp. 50-56.

13. Voir par exemple Malika Boudalia Greffou : L'école algérienne de Ibn Badis à Pavlov, Alger, 1989.

\section{RÉSUMÉS}

On trouvera dans cet article une présentation rapide du concept de français langue seconde (FLS), avec sa définition et son positionnement par rapport aux concepts voisins de français langue maternelle (FLM) et de français langue étrangère (FLE), puis une discussion da la question $d u$ français langue seconde vis-à-vis du français pour migrants. On essaiera enfin de répondre à ceux qui s'interrogent sur l'utilité même d'un tel concept en didactique du FLE.

One will find in this article, a quick overview of the concept of french as a second language (FSL), with that term s definition and its position vis-à-vis the closely related concepts of french as a mother tongue (FMT) and french as a foreign language (FFL), followed by a discussion of the issue of french as a second language versus that of french for immigrants. Lastly, the author endeavours to reply to those who raise questions as to the very use of such a concept in the didactics of french as a foreign language.

\section{INDEX}

Mots-clés : didactique des langues, français langue étrangère (FLE), français langue maternelle (FLM), français langue seconde (FLS), français pour migrants

Keywords : didactic of languages, french as a foreign language (FFL), french as a mother tongue (FMT), french as a second language (FSL), french for migrant population

\section{AUTEUR}

\section{JEAN-PIERRE CUQ}

Professeur à l'université Stendhal de Grenoble, directeur du Centre Universitaire d'études Françaises (CUEF) 